\title{
New variable selection methods for zero-inflated count data with applications to the substance abuse field
}

\author{
Anne Buu ${ }^{\mathrm{a} * \dagger}$, Norman J. Johnson ${ }^{\mathrm{b}}$, Runze $\mathrm{Li}^{\mathrm{c}}$ and Xianming Tan ${ }^{\mathrm{d}}$
}

\begin{abstract}
Zero-inflated count data are very common in health surveys. This study develops new variable selection methods for the zero-inflated Poisson regression model. Our simulations demonstrate the negative consequences which arise from the ignorance of zero-inflation. Among the competing methods, the one-step SCAD method is recommended because it has the highest specificity, sensitivity, exact fit, and lowest estimation error. The design of the simulations is based on the special features of two large national databases commonly used in the alcoholism and substance abuse field so that our findings can be easily generalized to the real settings. Applications of the methodology are demonstrated by empirical analyses on the data from a well-known alcohol study. Copyright (c) 2011 John Wiley \& Sons, Ltd.
\end{abstract}

Keywords: LASSO; one-step SCAD; variable selection; zero-inflated Poisson distribution

\section{Introduction}

Health surveys commonly inquire about participants' symptoms of target diseases. The resulting symptom count is an important indicator of the severity of a particular disease. Identifying risk factors for a disease can provide invaluable guidance for policy making and prevention programming. Our methodological research has been motivated by the challenges we encountered when building a multilevel model of individual, familial, and neighborhood influences on the symptomatology of alcohol use disorder (AUD). Although being the most distal among the three levels of influence, many risk factors involving the neighborhood environment, such as high poverty rate and unemployment rate, have been found to be associated with residents' alcohol or other substance use [1-5]. The neighborhood environment is usually characterized by descriptive statistics at the census tract level. Through geocoding, study participants' symptom count data and potential individual and familial risk factors can be merged with potential risk factors at the neighborhood level (i.e. the census tract level). However, there are many candidate variables in the census data and some of them are highly correlated. Our goal is to select a subset of important neighborhood risk factors for AUD symptomatology that can be used for model building purposes.

When health surveys are conducted on the general population or a community sample, the symptom count measure tends to have a high frequency of zero values. In the context of alcohol research, such excess zeros in the data come from nondrinkers or drinkers who have not developed AUD symptoms. Because zero-inflated count data are very common in health surveys, a statistical method that can model such highly skewed discrete distributions is highly desirable in practice. Classic variable

${ }^{a}$ Department of Psychiatry, University of Michigan, 4250 Plymouth Road, Ann Arbor, MI 48109, U.S.A.

${ }^{b}$ U.S. Census Bureau, Suitland, MD 20746, U.S.A.

${ }^{c}$ Department of Statistics and The Methodology Center, Pennsylvania State University, University Park, PA 16802, U.S.A.

${ }^{d}$ The Methodology Center, Pennsylvania State University, University Park, PA 16802, U.S.A.

*Correspondence to: Anne Buu, Department of Psychiatry, University of Michigan, 4250 Plymouth Road, Ann Arbor, MI 48109, U.S.A.

${ }^{\dagger}$ E-mail: buu@umich.edu 
selection criteria (e.g. AIC [6] and BIC [7]) and traditional variable selection procedures, including stepwise and best subset selection, may be adapted to the analysis of zero-inflated count data. However, traditional variable selection procedures are unstable - that is, small changes in the data may result in very different models [8]. Furthermore, when the pool of candidate variables is large, the best subset selection procedure becomes infeasible since it is computationally expensive.

Variable selection has been an active research area in the recent statistical literature. The Least Absolute Shrinkage and Selection Operator (LASSO) [9] and the Smoothly Clipped Absolute Deviation penalty (SCAD) [10] are two well-known variable selection procedures developed in the past decade. Both methods have desirable properties and both have been extended to generalized linear models that can handle binary, categorical, and count data [11,12]. The aim of this paper is to develop new variable selection procedures for the zero-inflated Poisson regression model (ZIP) [13] using LASSO and one-step SCAD techniques. In order to better assess the applicability of these new variable selection methods in the area of alcoholism and substance abuse research, we conduct simulations to evaluate their performance based on the data features of the U.S. census and a national health survey on alcohol and related conditions. We also demonstrate the use of our methodology by analyzing data from a well-known alcohol study.

This paper is organized as follows. In Section 2, we develop new variable selection methods for the ZIP model using LASSO and one-step SCAD techniques and we address issues related to the practical implementation of the proposed procedures. In Section 3, we conduct simulation studies to assess the performance of the proposed procedures, and investigate the impact of the ignorance of zero-inflation. In Section 4, we conduct an empirical analysis on the data from a community sample using the proposed procedures. Discussion and concluding remarks are presented in Section 5. The technical details and key derivation of the statistical property related to the proposed methods are given in the appendices.

\section{The model and variable selection methods}

\subsection{Zero-inflated Poisson regression model}

Suppose that $\left\{\boldsymbol{w}_{i}, y_{i}\right\}, i=1, \cdots, n$, is an independent and identically distributed sample from a population $(\boldsymbol{w}, y\}$. Let $\boldsymbol{x}_{i}$ and $z_{i}$ be $d_{1^{-}}$and $d_{2}$-dimensional sub-vectors of $\boldsymbol{w}_{i}$, respectively. Here $\boldsymbol{x}_{i}$ and $z_{i}$ may contain the same elements. Conditioning on $\boldsymbol{w}_{i}, y_{i}$ follows a zero-inflated Poisson (ZIP) distribution

$$
y_{i} \sim \pi_{i} \text { Poisson }(0)+\left(1-\pi_{i}\right) \operatorname{Poisson}\left(\lambda_{i}\right),
$$

where $\pi_{i}=\exp \left(z_{i}^{\prime} \gamma\right) /\left\{1+\exp \left(z_{i}^{\prime} \gamma\right)\right\}$ and $\lambda_{i}=\exp \left(\boldsymbol{x}_{i}^{\prime} \boldsymbol{\beta}\right)$ with unknown regression coefficient vectors $\boldsymbol{\beta}=$ $\left(\beta_{1}, \ldots, \beta_{d_{1}}\right)^{\prime}$ and $\gamma=\left(\gamma_{1}, \ldots, \gamma_{d_{2}}\right)^{\prime}$. Here Poisson $(0)$ stands for a degenerate distribution with the support point at 0 . To include an intercept, we set $x_{i 1}=1$ and $z_{i 1}=1$. Thus, $\beta_{1}$ and $\gamma_{1}$ are the corresponding intercepts. Model (1) is referred to as the ZIP model. From (1), the conditional probability mass function for $y_{i}$ is

$$
P\left(y_{i}=0 \mid \boldsymbol{w}_{i}\right)=\pi_{i}+\left(1-\pi_{i}\right) \mathrm{e}^{-\lambda_{i}} ; \quad P\left(y_{i}=m \mid \boldsymbol{w}_{i}\right)=\left(1-\pi_{i}\right) \mathrm{e}^{-\lambda_{i}} \lambda_{i}^{m} / m ! \quad \text { for } m=1,2, \ldots
$$

The logarithm of the likelihood function is

$$
\begin{aligned}
\ell(\boldsymbol{\beta}, \boldsymbol{\gamma})= & \sum_{y_{i}=0} \log \left[\exp \left(z_{i}^{\prime} \gamma\right)+\exp \left\{-\exp \left(\boldsymbol{x}_{i}^{\prime} \boldsymbol{\beta}\right)\right\}\right]+\sum_{y_{i}>0}\left\{y_{i} \boldsymbol{x}_{i}^{\prime} \boldsymbol{\beta}-\exp \left(\boldsymbol{x}_{i}^{\prime} \boldsymbol{\beta}\right)\right\} \\
& -\sum_{i=1}^{n} \log \left\{1+\exp \left(z_{i}^{\prime} \gamma\right)\right\}-\sum_{y_{i}>0} \log \left(y_{i} !\right) .
\end{aligned}
$$

Lambert [13] proposed the ZIP to model zero-inflated count data collected from a quality control study, in which the response typically is the number of defective products in a sample unit. The major strength of the ZIP model is that it can simultaneously accommodate one set of factors $\boldsymbol{x}_{i}$ that contribute to fewer defects in the imperfect state and another set of factors $z_{i}$ that make the perfect state more likely. The model has been applied in many fields including medicine (e.g. [14]). An alternative model, the hurdle model [15], originated in the economic literature that postulates a two-stage decision structure in the demand process: the first stage involves a selection process leading to zero or non-zero outcomes (a logit model); the second stage models the distribution of non-zero outcomes (a truncated Poisson model). The ZIP model is more intuitive when the population consists of a group of people 


\section{Statistics}

who can only have zero symptoms (e.g. nondrinkers) and another group who may have zero symptoms (e.g. drinkers). Thus, the logic behind the ZIP model fits better with our practical setting that does not involve a clear choice between zero and non-zero outcomes (symptoms). For this reason, we adopted the ZIP model in this paper.

\subsection{Variable selection}

Health surveys commonly collect many variables that can potentially be included in the model. In practice, it is desirable to select important variables and have a parsimonious model in order to improve prediction accuracy and model interpretability [9]. Here we propose new variable selection procedures for the ZIP model using the penalized likelihood method.

The penalized likelihood for the ZIP model is defined to be

$$
Q(\boldsymbol{\beta}, \boldsymbol{\gamma})=\ell(\boldsymbol{\beta}, \boldsymbol{\gamma})-n \sum_{j=1}^{d_{1}} p_{a_{j}}\left(\left|\beta_{j}\right|\right)-n \sum_{k=1}^{d_{2}} p_{b_{k}}\left(\left|\gamma_{k}\right|\right)
$$

where $p_{a_{j}}(\cdot)$ and $p_{b_{k}}(\cdot)$ are penalty functions with tuning parameters $a_{j}$ and $b_{k}$. The regression coefficients, $\beta_{j}$ and $\gamma_{k}$, are allowed to have different penalties. In particular, we may set $p_{a_{1}}\left(\left|\beta_{1}\right|\right)=p_{b_{1}}\left(\left|\gamma_{1}\right|\right)=$ 0 in order not to penalize the intercepts $\beta_{1}$ and $\gamma_{1}$. Fan and Li [10] studied the choice of penalty function in depth. In this paper, we consider only the most commonly used penalties developed in the recent literature: the $L_{1}$ penalty, defined by $p_{\tau}(\alpha)=\tau|\alpha|$, and the SCAD penalty, defined by

$$
p_{\tau}(|\alpha|)= \begin{cases}\tau|\alpha| & \text { if } 0 \leqslant|\alpha|<\tau \\ -\left(|\alpha|^{2}-2 c \tau|\alpha|+\tau^{2}\right) /[2(c-1)] & \text { if } \tau \leqslant|\alpha|<c \tau \\ (c+1) \tau^{2} / 2 & \text { if }|\alpha| \geqslant c \tau,\end{cases}
$$

where the value of $c=3.7$, as suggested in Fan and $\mathrm{Li}[10]$.

For linear regression models, the penalized least squares with the $L_{1}$ penalty leads to the LASSO proposed by Tibshirani [9]. The advantage of the penalized least squares with the $L_{1}$ penalty is that the entire solution path of the LASSO estimator can be constructed using the Least Angle Regression (LAR) [16]. As demonstrated in Fan and Li [10], the penalized least squares with the SCAD penalty possess good theoretical properties, particularly the oracle property (i.e. the resulting estimator asymptotically performs as well as if we knew the true submodel). For this reason, we consider only the $L_{1}$ and SCAD penalties.

The likelihood function for the ZIP model is, however, much more complicated than the leastsquares function for linear regression models or the likelihood function for generalized linear models. To maximize the penalized likelihood function (3), we adapt the one-step sparse estimator strategy proposed in Zou and Li [12].

Set the initial values $\left(\boldsymbol{\beta}^{(0)}, \boldsymbol{\gamma}^{(0)}\right)$ to be the un-penalized maximum likelihood estimates $(\hat{\boldsymbol{\beta}}, \hat{\boldsymbol{\gamma}})$. The $\log$-likelihood function $\ell(\boldsymbol{\beta}, \boldsymbol{\gamma})$ can be locally approximated by

$$
\ell\left(\boldsymbol{\beta}^{(0)}, \boldsymbol{\gamma}^{(0)}\right)+\frac{1}{2}\left[\left(\boldsymbol{\beta}-\boldsymbol{\beta}^{(0)}\right)^{\prime},\left(\boldsymbol{\gamma}-\boldsymbol{\gamma}^{(0)}\right)^{\prime}\right]\left[\nabla^{2} \ell\left(\boldsymbol{\beta}^{(0)}, \boldsymbol{\gamma}^{(0)}\right)\right]\left[\begin{array}{c}
\boldsymbol{\beta}-\boldsymbol{\beta}^{(0)} \\
\boldsymbol{\gamma}-\boldsymbol{\gamma}^{(0)}
\end{array}\right],
$$

where $\nabla^{2} \ell\left(\boldsymbol{\beta}^{(0)}, \boldsymbol{\gamma}^{(0)}\right)$ is the Hessian matrix of the log-likelihood function, since the gradient of the $\log$-likelihood function at the initial value $\nabla \ell\left(\boldsymbol{\beta}^{(0)}, \boldsymbol{\gamma}^{(0)}\right)=0$. The penalty functions can be locally linear approximated by

$$
p_{a_{j}}\left(\left|\beta_{j}\right|\right) \approx p_{a_{j}}\left(\left|\beta_{j}^{(0)}\right|\right)+p_{a_{j}}^{\prime}\left(\left|\beta_{j}^{(0)}\right|\right)\left(\left|\beta_{j}\right|-\left|\beta_{j}^{(0)}\right|\right) \quad \text { for } \beta_{j} \approx \beta_{j}^{(0)}
$$

and

$$
p_{b_{k}}\left(\left|\gamma_{k}\right|\right) \approx p_{b_{k}}\left(\left|\gamma_{k}^{(0)}\right|\right)+p_{b_{k}}^{\prime}\left(\left|\gamma_{k}^{(0)}\right|\right)\left(\left|\gamma_{k}\right|-\left|\gamma_{k}^{(0)}\right|\right) \text { for } \gamma_{k} \approx \gamma_{k}^{(0)}
$$


Thus, the one-step sparse estimator for the ZIP model is defined to be

$$
\begin{aligned}
& \left(\hat{\boldsymbol{\beta}}^{(1)}, \hat{\boldsymbol{\gamma}}^{(1)}\right)=\arg \min _{\boldsymbol{\beta}, \boldsymbol{\gamma}}\left\{\frac{1}{2}\left[\left(\boldsymbol{\beta}-\boldsymbol{\beta}^{(0)}\right)^{\prime},\left(\boldsymbol{\gamma}-\boldsymbol{\gamma}^{(0)}\right)^{\prime}\right]\left[-\nabla^{2} \ell\left(\boldsymbol{\beta}^{(0)}, \boldsymbol{\gamma}^{(0)}\right)\right]\left[\begin{array}{c}
\boldsymbol{\beta}-\boldsymbol{\beta}^{(0)} \\
\boldsymbol{\gamma}-\boldsymbol{\gamma}^{(0)}
\end{array}\right]\right. \\
& \left.+n \sum_{j=1}^{d_{1}} p_{a_{j}}^{\prime}\left(\left|\beta_{j}^{(0)}\right|\right)\left|\beta_{j}\right|+n \sum_{k=1}^{d_{2}} p_{b_{k}}^{\prime}\left(\left|\gamma_{k}^{(0)}\right|\right)\left|\gamma_{k}\right|\right\} .
\end{aligned}
$$

When the SCAD penalty is employed, this one-step sparse estimator is referred to as one-step SCAD that can be viewed as an adaptive LASSO with weights obtained from the SCAD penalty. Since the first term in the objective function is a quadratic function of $\left(\boldsymbol{\beta}^{\prime}, \gamma^{\prime}\right)$, and the penalty function is a weighted $L_{1}$ penalty, we employ the LARS algorithm to obtain the one-step sparse estimator. See Appendix A for the technical details related to the implementation of the LARS algorithm. Using the same techniques employed in Zou and Li [12], it can be shown that the one-step SCAD possesses an oracle property (see Appendix B for the key derivation). The local quadratic approximation (4) of the logarithm of the likelihood function indeed is the same as the least-squares approximation in Wang and Leng [17], in which the authors emphasized the adaptive LASSO penalty in comparison to the SCAD penalty used in this article.

Automatic selection of the tuning parameters $a_{j}$ and $b_{k}$ using data-driven methods is desirable and yet computationally expensive because one has to search over a $\left(d_{1}+d_{2}\right)$-dimensional grid for the proposed one-step sparse estimator. To save computation cost, we follow the strategy of Fan and Li [18] and set $a_{j}=\tau \operatorname{SE}\left(\hat{\beta}_{j}^{(0)}\right)$ and $b_{k}=\tau \operatorname{SE}\left(\hat{\gamma}_{k}^{(0)}\right)$, where $\operatorname{SE}\left(\hat{\beta}_{j}^{(0)}\right)$ and $\operatorname{SE}\left(\hat{\gamma}_{k}^{(0)}\right)$ are the standard errors of the unpenalized maximum likelihood estimate of $\beta_{j}$ and $\gamma_{k}$, respectively. This procedure reduces the search for $\tau$ to a set of one-dimensional grid points. In our simulation studies and our empirical analysis using a real data set, $\tau$ is determined from a modification of the BIC tuning parameter selector [19]. Our simulation results show that this strategy for determining the tuning parameters works well.

\section{Simulation study}

Most simulation studies in the variable selection literature employ covariates that are idealistically distributed (e.g. multivariate normal) and parameters that are arbitrarily determined. However, as pointed out by Burton et al. [20], simulated data should closely represent the structure of real data so that the results can be generalizable to real situations and thus have credibility. One unique strength of this study is that our simulation experiments are based on the special features of two large national databases: the 2000 U.S. census and the National Epidemiologic Survey on Alcohol and Related Conditions (NESARC) [21]. For this reason, our results can be used to guide future applications of the proposed methods in the field of alcoholism and substance abuse research. In Experiment 1, we used a census database as a pseudo-population from which to draw covariate values in varied sample sizes. We determined parameter values by fitting a ZIP model to census and NESARC data. In order to evaluate the performance of competing methods under different correlation structures, we conducted Experiment 2 that employed the same set of parameter values as in Experiment 1 but drew random samples from multivariate normal distributions with varied levels of correlation and sample sizes. In Experiment 3, the other two factors were manipulated while holding the correlation and sample size at the medium values: the proportion of non-zero coefficients and the proportion of zero outcome.

\subsection{Experiment 1: sampling from census data with varied sample sizes}

This experiment aims to evaluate the performance of the following four competing methods, when the true model is a ZIP model with two different sets of covariates in the Poisson component and the zero component:

1. Poisson regression with LASSO (PR-LASSO)

2. Poisson regression with SCAD (PR-SCAD)

3. ZIP regression with LASSO (ZIP-LASSO)

4. ZIP regression with SCAD (ZIP-SCAD). 


\begin{tabular}{|ll|}
\hline Table I. The covariates derived from the 2000 U.S. census data. \\
1 & Proportion of male residents \\
2 & Proportion of residents aged 25+ who dropped out of high school \\
3 & Proportion of residents aged 16+ who are unemployed \\
4 & Proportion of residents aged 16+ with professional or managerial occupations \\
5 & Proportion of residents aged 16+ who are not in labor force \\
6 & Resident per capita income \\
7 & Proportion of residents with public assistance income in 1999 \\
8 & Proportion of households with public assistance income in 1999 \\
9 & Proportion of residents under poverty line in 1999 \\
10 & Proportion of residents who are black \\
11 & Proportion of residents who are Hispanic or Latino \\
12 & Proportion of residents born outside the U.S. \\
13 & Proportion of households with husband, wife and children under age 18 \\
14 & Proportion of households that are female-headed and have children under age 18 \\
15 & Average household size \\
16 & Proportion of housing units not owned by occupants \\
17 & Proportion of vacant housing \\
18 & Proportion of residents aged 5+ who did not live at the same address 5 years earlier \\
20 & Proportion of disabled residents aged 5+ \\
\hline
\end{tabular}

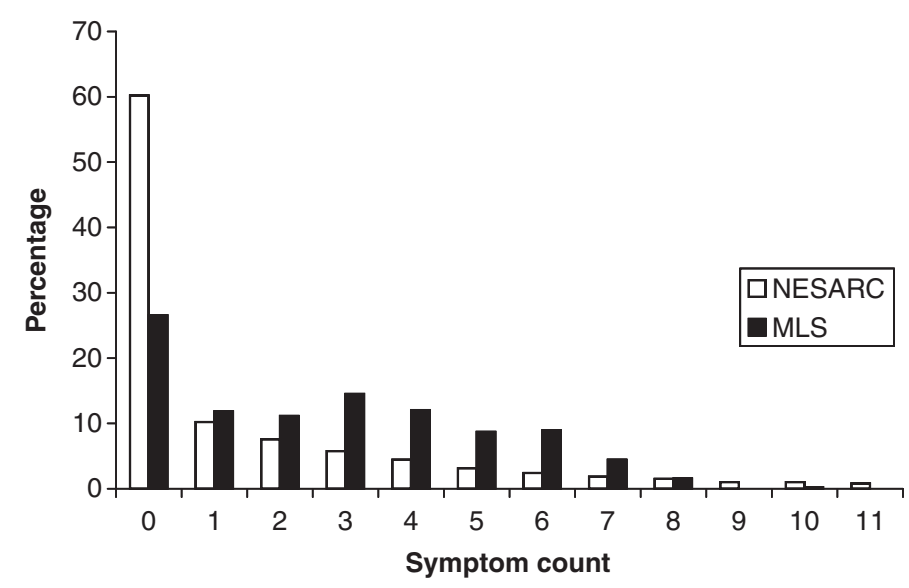

NESARC: National Epidemiologic Survey on Alcohol and Related Conditions; MLS: Michigan Longitudinal Study

Figure 1. Distributions of DSM-IV AUD symptom counts.

In order to construct covariates with real data properties, we obtained the statistical characteristics of 66304 census tracts from the official 2000 U.S. census Web site and from these derived the 20 composite variables which have been used in the substance abuse literature to indicate neighborhood risk (listed in Table I). These variables were standardized (with sample means and standard deviations) and used as candidates for variable selection in the Poisson and zero components of the model. Not surprisingly, some of these variables are highly correlated (Pearson's $r=0.01-0.80$ ). By combining these covariates along with the AUD symptom counts from the NESARC database (i.e. the outcome), we were able to conduct exploratory analyses and determine the following set of true parameter values:

$$
\begin{aligned}
& \boldsymbol{\beta}=(1.10,0,0,0,-0.36,0,0,0,0,0,0,0,0,-0.32,0,0,0,0,0,0,0)^{\prime} \\
& \gamma=(0.30,-0.48,0,0,0,0.40,0,0,0,0,0.44,0,0.44,0,0,0,0,0,0,0,0)^{\prime} .
\end{aligned}
$$

Applying this set of ZIP regression parameters to the covariates $\boldsymbol{x}_{i}\left(=z_{i}\right)$ from the $i$ th census tract, we generated the outcome $Y_{i}(i=1, \ldots, 66304)$. The resulting outcome distributes like the AUD symptom count in the NESARC database (see Figure 1). We randomly drew a sample of size $n$ from the resulting 66304 covariates-outcome pairs and applied the four statistical methods to analyze the data. 


\begin{tabular}{|c|c|c|c|c|c|c|c|c|}
\hline \multirow[b]{2}{*}{ Method } & \multirow[b]{2}{*}{ MRMSE* } & \multirow[b]{2}{*}{ Specificity ${ }^{\dagger}$} & \multirow[b]{2}{*}{ Sensitivity $\ddagger$} & \multirow[b]{2}{*}{ Under $\mathrm{fit}^{\S}$} & \multirow[b]{2}{*}{ Exact fit ${ }^{\mathbb{I}}$} & \multicolumn{3}{|c|}{ Over fit $\|$} \\
\hline & & & & & & 1 & 2 & $\geqslant 3$ \\
\hline \multicolumn{9}{|c|}{$\begin{array}{l}\text { The Poisson component } \\
n=300\end{array}$} \\
\hline PR-LASSO & 0.923 & 0.465 & 0.771 & 0.419 & 0.000 & 0.000 & 0.000 & 0.581 \\
\hline PR-SCAD & 1.716 & 0.575 & 0.686 & 0.532 & 0.000 & 0.000 & 0.003 & 0.465 \\
\hline ZIP-LASSO & 0.218 & 0.808 & 0.760 & 0.366 & 0.010 & 0.050 & 0.103 & 0.471 \\
\hline $\begin{array}{l}\text { ZIP-SCAD } \\
n=600\end{array}$ & 0.201 & 0.891 & 0.740 & 0.452 & 0.154 & 0.163 & 0.118 & 0.113 \\
\hline PR-LASSO & 2.060 & 0.425 & 0.921 & 0.157 & 0.000 & 0.000 & 0.000 & 0.843 \\
\hline PR-SCAD & 3.112 & 0.581 & 0.858 & 0.273 & 0.000 & 0.000 & 0.000 & 0.727 \\
\hline ZIP-LASSO & 0.336 & 0.774 & 0.973 & 0.050 & 0.010 & 0.044 & 0.142 & 0.754 \\
\hline ZIP-SCAD & 0.101 & 0.903 & 0.964 & 0.073 & 0.247 & 0.279 & 0.197 & 0.204 \\
\hline \multicolumn{9}{|c|}{$\begin{array}{l}\text { The zero component } \\
n=300\end{array}$} \\
\hline ZIP-LASSO & 0.280 & 1.000 & 0.002 & 1.000 & 0.000 & 0.000 & 0.000 & 0.000 \\
\hline $\begin{array}{l}\text { ZIP-SCAD } \\
n=600\end{array}$ & 0.210 & 0.983 & 0.315 & 0.978 & 0.008 & 0.010 & 0.004 & 0.000 \\
\hline ZIP-LASSO & 0.746 & 0.997 & 0.074 & 0.952 & 0.026 & 0.019 & 0.001 & 0.002 \\
\hline ZIP-SCAD & 0.292 & 0.981 & 0.670 & 0.785 & 0.159 & 0.046 & 0.010 & 0.000 \\
\hline
\end{tabular}

${ }^{*}$ MRMSE $=$ Median of the ratio of the reduced model MSE to the full model MSE.

$\dagger_{\text {Specificity }}=$ Mean of the proportion of zero coefficients that were correctly identified.

$\ddagger$ Sensitivity $=$ Mean of the proportion of nonzero coefficients that were correctly identified.

${ }^{\S}$ Under fit $=$ Probability of excluding any significant coefficients.

IExact fit $=$ Probability of selecting the exact sub-model.

$\|_{\text {Over }}$ fit $=$ Probabilities of including all significant variables and some noise variables $(1,2, \geqslant 3)$.

Three sample sizes, small $(n=300)$, medium $(n=600)$, and large $(n=900)$, were chosen based on our survey of existing studies in the substance abuse field (e.g. [22-27]). This experiment was replicated 1000 times.

In Table II, the performance of the four competing statistical methods are evaluated based on several criteria. For each replication, we computed the mean squared error (MSE) for both the reduced model and the full ZIP model, then computed the ratio of these two MSE values. The median of the ratios from 1000 replications is reported under the column 'MRMSE.' A smaller value indicates a better performance in terms of parameter estimation. We also calculated the specificity and sensitivity for each replication. Specificity is defined as the proportion of zero coefficients that were correctly estimated to be zero; sensitivity is the proportion of nonzero coefficients that were correctly estimated to be nonzero. The averages of both indices over 1000 replications are listed under the columns with corresponding headings in the table. 'Under fit' is defined as the probability of excluding any significant coefficients in 1000 replications, whereas 'exact fit' is the probability of selecting the exact sub-model. The probabilities of including all significant variables and some noise variables $(1,2, \geqslant 3)$ are also reported in the columns under 'over fit.'

Table II summarizes the simulation results for the Poisson component (top of table) and the zero component (bottom of table) of the ZIP model. In order to save space, we omit the results of the $n=900$ condition from the table. Interested readers may request the technical report with a complete table from the first author. For the Poisson component, the Poisson regression methods (i.e. PR-LASSO and PR-SCAD) tended to have higher values of MRMSE, low specificity, and never fit the model exactly because they did not take into account the excess zeros in the data. Their lower levels of specificity $(0.4-0.6)$ stemmed from their greater tendency to over fit the model (i.e. select noise variables). Between the ZIP regression methods, the SCAD outperformed the LASSO on MRMSE, specificity, and exact fit across all sample sizes. ZIP-LASSO tended to over fit to a large degree and thus performed poorly. In terms of the zero component, we only compared the two ZIP methods because the Poisson regression methods did not employ the zero component. ZIP-SCAD outperformed ZIP-LASSO on MRMSE, sensitivity, and exact fit across all sample sizes. The performance of both methods on sensitivity and exact fit tended to improve as the sample size increased. 


\subsection{Experiment 2: manipulating correlations and sample sizes}

In Experiment 1, we drew random samples from the census data that served as a pseudo-population having a given correlation structure. In order to evaluate the performance of the competing methods under different correlation structures, we conducted Experiment 2 by manipulating the levels of correlation while employing the same set of parameter values and sample sizes as in Experiment 1 . We drew random samples from a multivariate normal distribution $N_{20}(\mathbf{0}, \Sigma)$, where the diagonal element $\sigma_{i i}=1$ and the off-diagonal element $\sigma_{i j}=\rho^{|i-j|}(i, j=1, \ldots, 20)$. Because the correlation coefficients for the covariates in census data range from 0.01 to 0.80 , three levels of correlation were used in this experiment: small $(\rho=0)$, medium $(\rho=0.4)$, and large $(\rho=0.8)$.

The results of Experiment 2 are depicted in Tables III and IV. In order to save space, we omit the results of the $n=900$ and $\rho=0.8$ conditions from the tables. Interested readers may request the technical report with complete tables from the first author. Some of the general trends observed in Experiment 1 are also observed in Experiment 2. The Poisson regression methods had high MRMSE, low specificity, and zero exact fit across the three levels of correlation due to their tendency to over fit the model. Between the two ZIP methods, the SCAD tended to have lower MRMSE in both the Poisson and the zero components, higher sensitivity in the zero component, and higher exact fit in the zero component. As the sample size increased or the correlation decreased, the ZIP-SCAD had a noticeable improvement in performance in the zero component. The ZIP-LASSO's performance, on the other hand, followed a clear pattern of improvement in the Poisson component as the correlation decreased.

\subsection{Experiment 3: manipulating proportions of non-zero coefficients and proportions of zero outcome}

In Experiment 3, we evaluated the performance of the competing methods when the proportion of non-zero coefficients and the proportion of zero outcome were varied. Since the effects of correlation and sample size on the performance were tested in Experiment 2, we fixed both factors at their medium values: $\rho=0.4, n=600$. The proportion of non-zero coefficients was manipulated at three levels: 15 ,

\begin{tabular}{|c|c|c|c|c|c|c|c|c|}
\hline \multirow[b]{2}{*}{ Method } & \multirow[b]{2}{*}{ MRMSE* } & \multirow[b]{2}{*}{ Specificity $^{\dagger}$} & \multirow[b]{2}{*}{ Sensitivity ${ }^{\ddagger}$} & \multirow[b]{2}{*}{ Under $\mathrm{fit}^{\S}$} & \multirow[b]{2}{*}{ Exactfit $^{\text {Il }}$} & \multicolumn{3}{|c|}{ Over fit ${ }^{\|}$} \\
\hline & & & & & & 1 & 2 & $\geqslant 3$ \\
\hline \multicolumn{9}{|c|}{$\begin{array}{l}\text { The Poisson component } \\
n=300\end{array}$} \\
\hline PR-LASSO & 2.421 & 0.477 & 0.994 & 0.012 & 0.001 & 0.001 & 0.006 & 0.980 \\
\hline PR-SCAD & 3.321 & 0.609 & 0.988 & 0.025 & 0.002 & 0.007 & 0.012 & 0.954 \\
\hline ZIP-LASSO & 0.375 & 0.955 & 0.996 & 0.008 & 0.502 & 0.286 & 0.124 & 0.080 \\
\hline $\begin{array}{l}\text { ZIP-SCAD } \\
n=600\end{array}$ & 0.094 & 0.971 & 0.999 & 0.002 & 0.664 & 0.208 & 0.084 & 0.042 \\
\hline PR-LASSO & 5.381 & 0.479 & 1.000 & 0.000 & 0.000 & 0.000 & 0.000 & 1.000 \\
\hline PR-SCAD & 6.932 & 0.641 & 1.000 & 0.001 & 0.000 & 0.000 & 0.000 & 0.999 \\
\hline ZIP-LASSO & 0.508 & 0.968 & 1.000 & 0.000 & 0.617 & 0.247 & 0.094 & 0.042 \\
\hline ZIP-SCAD & 0.154 & 0.921 & 1.000 & 0.000 & 0.288 & 0.304 & 0.223 & 0.185 \\
\hline \multicolumn{9}{|c|}{$\begin{array}{l}\text { The zero component } \\
n=300\end{array}$} \\
\hline ZIP-LASSO & 1.470 & 1.000 & 0.000 & 1.000 & 0.000 & 0.000 & 0.000 & 0.000 \\
\hline $\begin{array}{l}\text { ZIP-SCAD } \\
n=600\end{array}$ & 1.192 & 1.000 & 0.185 & 0.983 & 0.015 & 0.002 & 0.000 & 0.000 \\
\hline ZIP-LASSO & 3.516 & 1.000 & 0.001 & 1.000 & 0.000 & 0.000 & 0.000 & 0.000 \\
\hline ZIP-SCAD & 0.642 & 0.998 & 0.793 & 0.516 & 0.462 & 0.020 & 0.002 & 0.000 \\
\hline
\end{tabular}

${ }^{*}$ MRMSE $=$ Median of the ratio of the reduced model MSE to the full model MSE.

$\dagger$ Specificity = Mean of the proportion of zero coefficients that were correctly identified.

${ }^{\ddagger}$ Sensitivity $=$ Mean of the proportion of nonzero coefficients that were correctly identified.

$\S$ Under fit $=$ Probability of excluding any significant coefficients.

$\mathbb{I}$ Exact fit $=$ Probability of selecting the exact sub-model.

$\|_{\text {Over fit }}=$ Probabilities of including all significant variables and some noise variables $(1,2, \geqslant 3)$. 


\begin{tabular}{|c|c|c|c|c|c|c|c|c|}
\hline \multirow[b]{2}{*}{ Method } & \multirow[b]{2}{*}{ MRMSE* } & \multirow[b]{2}{*}{ Specificity ${ }^{\dagger}$} & \multirow[b]{2}{*}{ Sensitivity $\ddagger$} & \multirow[b]{2}{*}{ Under it $^{\S}$} & \multirow[b]{2}{*}{ Exact fit ${ }^{\mathbb{I l}}$} & \multicolumn{3}{|c|}{ Over fit $\|$} \\
\hline & & & & & & 1 & 2 & $\geqslant 3$ \\
\hline \multicolumn{9}{|c|}{$\begin{array}{l}\text { The Poisson component } \\
n=300\end{array}$} \\
\hline PR-LASSO & 1.965 & 0.521 & 0.997 & 0.007 & 0.000 & 0.000 & 0.003 & 0.990 \\
\hline PR-SCAD & 2.698 & 0.629 & 0.986 & 0.028 & 0.000 & 0.005 & 0.027 & 0.940 \\
\hline ZIP-LASSO & 0.281 & 0.941 & 0.998 & 0.004 & 0.380 & 0.325 & 0.181 & 0.110 \\
\hline $\begin{array}{l}\text { ZIP-SCAD } \\
n=600\end{array}$ & 0.086 & 0.961 & 1.000 & 0.000 & 0.570 & 0.251 & 0.109 & 0.070 \\
\hline PR-LASSO & 4.107 & 0.517 & 1.000 & 0.000 & 0.000 & 0.000 & 0.000 & 1.000 \\
\hline PR-SCAD & 5.388 & 0.637 & 1.000 & 0.001 & 0.000 & 0.000 & 0.003 & 0.996 \\
\hline ZIP-LASSO & 0.361 & 0.956 & 1.000 & 0.000 & 0.494 & 0.310 & 0.139 & 0.057 \\
\hline ZIP-SCAD & 0.125 & 0.909 & 1.000 & 0.000 & 0.234 & 0.291 & 0.229 & 0.246 \\
\hline \multicolumn{9}{|c|}{$\begin{array}{l}\text { The zero component } \\
n=300\end{array}$} \\
\hline ZIP-LASSO & 1.103 & 1.000 & 0.000 & 1.000 & 0.000 & 0.000 & 0.000 & 0.000 \\
\hline $\begin{array}{l}\text { ZIP-SCAD } \\
n=600\end{array}$ & 0.902 & 0.999 & 0.224 & 0.973 & 0.026 & 0.001 & 0.000 & 0.000 \\
\hline ZIP-LASSO & 2.673 & 1.000 & 0.000 & 1.000 & 0.000 & 0.000 & 0.000 & 0.000 \\
\hline ZIP-SCAD & 0.536 & 0.997 & 0.781 & 0.557 & 0.411 & 0.028 & 0.003 & 0.001 \\
\hline
\end{tabular}

${ }^{*}$ MRMSE $=$ Median of the ratio of the reduced model MSE to the full model MSE.

${ }^{\dagger}$ Specificity $=$ Mean of the proportion of zero coefficients that were correctly identified.

$\ddagger$ Sensitivity $=$ Mean of the proportion of nonzero coefficients that were correctly identified.

$\S$ Under fit $=$ Probability of excluding any significant coefficients.

IExact fit $=$ Probability of selecting the exact sub-model.

$\|_{\text {Over }}$ fit $=$ Probabilities of including all significant variables and some noise variables $(1,2, \geqslant 3)$.

30, and 45 per cent. The proportion of zero outcome was also varied at three levels: 30,45 , and 60 per cent. The following are the parameter values that generated these $3 \times 3$ settings:

15 per cent non-zero coefficients with 30 per cent zero outcome

$$
\begin{aligned}
& \boldsymbol{\beta}=(1.10,0,0,0,-0.36,0,0,0,0,0,0,0,0,-0.32,0,0,0,0,0,0,0)^{\prime} \\
& \gamma=(-1.20,-0.48,0,0,0,0.40,0,0,0,0,0.44,0,0.44,0,0,0,0,0,0,0,0)^{\prime}
\end{aligned}
$$

15 per cent non-zero coefficients with 45 per cent zero outcome

$$
\begin{aligned}
& \boldsymbol{\beta}=(1.10,0,0,0,-0.36,0,0,0,0,0,0,0,0,-0.32,0,0,0,0,0,0,0)^{\prime} \\
& \gamma=(-0.35,-0.48,0,0,0,0.40,0,0,0,0,0.44,0,0.44,0,0,0,0,0,0,0,0)^{\prime}
\end{aligned}
$$

15 per cent non-zero coefficients with 60 per cent zero outcome

$$
\begin{aligned}
& \boldsymbol{\beta}=(1.10,0,0,0,-0.36,0,0,0,0,0,0,0,0,-0.32,0,0,0,0,0,0,0)^{\prime} \\
& \gamma=(0.30,-0.48,0,0,0,0.40,0,0,0,0,0.44,0,0.44,0,0,0,0,0,0,0,0)^{\prime}
\end{aligned}
$$

30 per cent non-zero coefficients with 30 per cent zero outcome

$$
\begin{aligned}
& \boldsymbol{\beta}=(1.50,0,0,0,-0.22,0,0,0,-0.25,0,0.20,0,0.30,-0.32,0,0,0,0.20,0,0,0)^{\prime} \\
& \gamma=(-1.05,0,0.45,0,-0.3,0,0,0,0,0,-0.33,0,-0.39,0,0,0.3,0,0,0,0.36,0)^{\prime}
\end{aligned}
$$

30 per cent non-zero coefficients with 45 per cent zero outcome

$$
\begin{aligned}
& \boldsymbol{\beta}=(1.10,0,0,0,-0.22,0,0,0,-0.25,0,0.20,0,0.30,-0.32,0,0,0,0.20,0,0,0)^{\prime} \\
& \gamma=(-0.41,0,0.45,0,-0.3,0,0,0,0,0,-0.33,0,-0.39,0,0,0.3,0,0,0,0.36,0)^{\prime}
\end{aligned}
$$


30 per cent non-zero coefficients with 60 per cent zero outcome

$$
\begin{aligned}
& \boldsymbol{\beta}=(0.10,0,0,0,-0.22,0,0,0,-0.25,0,0.20,0,0.30,-0.32,0,0,0,0.20,0,0,0)^{\prime} \\
& \gamma=(-0.3,0,0.45,0,-0.3,0,0,0,0,0,-0.33,0,-0.39,0,0,0.3,0,0,0,0.36,0)^{\prime}
\end{aligned}
$$

45 per cent non-zero coefficients with 30 per cent zero outcome

$$
\begin{aligned}
& \boldsymbol{\beta}=(1.8,0,0.28,0,-0.22,0,0,0,-0.25,0,0.20,0,0.30,-0.32,0,0.21,0,0.20,0,-0.28,0)^{\prime} \\
& \gamma=(-1.14,0,0.45,0,-0.3,0,-0.45,0,-0.54,0,-0.33,0,-0.39,0,0,0.3,0.6,0,0,0.36,0)^{\prime}
\end{aligned}
$$

45 per cent non-zero coefficients with 45 per cent zero outcome

$$
\begin{aligned}
& \boldsymbol{\beta}=(1.1,0,0.28,0,-0.22,0,0,0,-0.25,0,0.20,0,0.30,-0.32,0,0.21,0,0.20,0,-0.28,0)^{\prime} \\
& \gamma=(-0.6,0,0.45,0,-0.3,0,-0.45,0,-0.54,0,-0.33,0,-0.39,0,0,0.3,0.6,0,0,0.36,0)^{\prime}
\end{aligned}
$$

45 per cent non-zero coefficients with 60 per cent zero outcome

$$
\begin{aligned}
& \boldsymbol{\beta}=(0.5,0,0.28,0,-0.22,0,0,0,-0.25,0,0.20,0,0.30,-0.32,0,0.21,0,0.20,0,-0.28,0)^{\prime} \\
& \gamma=(-0.09,0,0.45,0,-0.3,0,-0.45,0,-0.54,0,-0.33,0,-0.39,0,0,0.3,0.6,0,0,0.36,0)^{\prime}
\end{aligned}
$$

Tables V and VI summarize the results of Experiment 3. In order to save space, we omit the results of the 60 per cent zeros and 45 per cent non-zero coefficient conditions from the table. Interested readers may request the technical report with complete tables from the first author. The Poisson regression methods performed poorly across all the nine settings due to their tendency to over fit the model. This

\begin{tabular}{|c|c|c|c|c|c|c|c|c|}
\hline \multirow[b]{2}{*}{ Method } & \multirow[b]{2}{*}{ MRMSE* } & \multirow[b]{2}{*}{ Specificity $^{\dagger}$} & \multirow[b]{2}{*}{ Sensitivity ${ }^{\ddagger}$} & \multirow[b]{2}{*}{ Under fit $^{\S}$} & \multirow[b]{2}{*}{ Exact fit ${ }^{\mathbb{I}}$} & \multicolumn{3}{|c|}{ Over fittl } \\
\hline & & & & & & 1 & 2 & $\geqslant 3$ \\
\hline \multicolumn{9}{|c|}{$\begin{array}{l}\text { The Poisson component } \\
30 \text { per cent zeros }\end{array}$} \\
\hline PR-LASSO & 1.344 & 0.655 & 1.000 & 0.000 & 0.000 & 0.001 & 0.026 & 0.973 \\
\hline PR-SCAD & 1.439 & 0.723 & 1.000 & 0.000 & 0.014 & 0.043 & 0.088 & 0.855 \\
\hline ZIP-LASSO & 0.391 & 0.961 & 1.000 & 0.000 & 0.538 & 0.290 & 0.121 & 0.051 \\
\hline ZIP-SCAD & 0.080 & 0.948 & 1.000 & 0.000 & 0.652 & 0.092 & 0.084 & 0.172 \\
\hline \multicolumn{9}{|c|}{45 per cent zeros } \\
\hline PR-LASSO & 2.864 & 0.568 & 1.000 & 0.000 & 0.000 & 0.000 & 0.001 & 0.999 \\
\hline PR-SCAD & 3.647 & 0.652 & 1.000 & 0.000 & 0.000 & 0.001 & 0.015 & 0.984 \\
\hline ZIP-LASSO & 0.372 & 0.953 & 1.000 & 0.000 & 0.479 & 0.316 & 0.126 & 0.079 \\
\hline ZIP-SCAD & 0.147 & 0.876 & 1.000 & 0.000 & 0.202 & 0.183 & 0.209 & 0.406 \\
\hline \multicolumn{9}{|c|}{$\begin{array}{l}\text { The zero component } \\
30 \text { per cent zeros }\end{array}$} \\
\hline ZIP-LASSO & 1.832 & 1.000 & 0.000 & 1.000 & 0.000 & 0.000 & 0.000 & 0.000 \\
\hline ZIP-SCAD & 1.508 & 1.000 & 0.192 & 0.950 & 0.049 & 0.001 & 0.000 & 0.000 \\
\hline \multicolumn{9}{|c|}{45 per cent zeros } \\
\hline ZIP-LASSO & 2.640 & 1.000 & 0.000 & 1.000 & 0.000 & 0.000 & 0.000 & 0.000 \\
\hline ZIP-SCAD & 0.794 & 0.998 & 0.655 & 0.680 & 0.309 & 0.009 & 0.002 & 0.000 \\
\hline
\end{tabular}
tendency was also observed in the other two experiments. While the two ZIP methods had comparable performance in the Poisson component, the ZIP-LASSO performed poorly (i.e. almost zero sensitivity and exact fit) across all the settings in the zero component. As the proportion of non-zero coefficients increased, the ZIP-LASSO's performance became worse in the Poisson component while the ZIPSCAD's performance was mostly affected in the zero component. When there were 15 or 30 per cent non-zero coefficients, the two ZIP methods tended to perform worse in the Poisson component as the proportion of zero outcome increased.

$*$ MRMSE $=$ Median of the ratio of the reduced model MSE to the full model MSE.

${ }^{\dagger}$ Specificity $=$ Mean of the proportion of zero coefficients that were correctly identified.

${ }^{\ddagger}$ Sensitivity = Mean of the proportion of nonzero coefficients that were correctly identified.

$\S_{\text {Under fit }}=$ Probability of excluding any significant coefficients.

$\mathbb{I}_{\text {Exact }}$ fit $=$ Probability of selecting the exact sub-model.

$\|_{\text {Over fit }}=$ Probabilities of including all significant variables and some noise variables $(1,2, \geqslant 3)$. 


\begin{tabular}{|c|c|c|c|c|c|c|c|c|}
\hline \multirow[b]{2}{*}{ Method } & \multirow[b]{2}{*}{ MRMSE* } & \multirow[b]{2}{*}{ Specificity $^{\dagger}$} & \multirow[b]{2}{*}{ Sensitivity ${ }^{\ddagger}$} & \multirow[b]{2}{*}{ Under $\mathrm{fit}^{\S}$} & \multirow[b]{2}{*}{ Exact fit ${ }^{\text {II }}$} & \multicolumn{3}{|c|}{ Over fit ${ }^{\|}$} \\
\hline & & & & & & 1 & 2 & $\geqslant 3$ \\
\hline \multicolumn{9}{|c|}{$\begin{array}{l}\text { The Poisson component } \\
30 \text { per cent zeros }\end{array}$} \\
\hline PR-LASSO & 4.088 & 0.437 & 1.000 & 0.000 & 0.000 & 0.000 & 0.004 & 0.996 \\
\hline PR-SCAD & 4.395 & 0.596 & 1.000 & 0.000 & 0.001 & 0.016 & 0.046 & 0.937 \\
\hline ZIP-LASSO & 0.936 & 0.828 & 1.000 & 0.000 & 0.101 & 0.214 & 0.245 & 0.440 \\
\hline ZIP-SCAD & 0.340 & 0.917 & 1.000 & 0.000 & 0.535 & 0.222 & 0.079 & 0.164 \\
\hline \multicolumn{9}{|c|}{45 per cent zeros } \\
\hline PR-LASSO & 4.024 & 0.438 & 0.988 & 0.070 & 0.000 & 0.000 & 0.002 & 0.928 \\
\hline PR-SCAD & 4.880 & 0.636 & 0.973 & 0.157 & 0.000 & 0.014 & 0.054 & 0.775 \\
\hline ZIP-LASSO & 0.903 & 0.818 & 1.000 & 0.000 & 0.085 & 0.221 & 0.237 & 0.457 \\
\hline ZIP-SCAD & 0.336 & 0.897 & 1.000 & 0.001 & 0.368 & 0.214 & 0.192 & 0.225 \\
\hline \multicolumn{9}{|c|}{$\begin{array}{l}\text { The zero component } \\
30 \text { per cent zeros }\end{array}$} \\
\hline ZIP-LASSO & 2.428 & 1.000 & 0.000 & 1.000 & 0.000 & 0.000 & 0.000 & 0.000 \\
\hline ZIP-SCAD & 2.256 & 0.999 & 0.100 & 0.993 & 0.007 & 0.000 & 0.000 & 0.000 \\
\hline \multicolumn{9}{|c|}{45 per cent zeros } \\
\hline ZIP-LASSO & 2.529 & 1.000 & 0.000 & 1.000 & 0.000 & 0.000 & 0.000 & 0.000 \\
\hline ZIP-SCAD & 1.477 & 0.997 & 0.357 & 0.961 & 0.035 & 0.004 & 0.000 & 0.000 \\
\hline
\end{tabular}

${ }^{*}$ MRMSE $=$ Median of the ratio of the reduced model MSE to the full model MSE.

$\dagger_{\text {Specificity }}=$ Mean of the proportion of zero coefficients that were correctly identified.

${ }_{\text {Sensitivity }}=$ Mean of the proportion of nonzero coefficients that were correctly identified.

${ }^{\S}$ Under fit $=$ Probability of excluding any significant coefficients.

IExact fit $=$ Probability of selecting the exact sub-model.

$\|_{\text {Over }}$ fit $=$ Probabilities of including all significant variables and some noise variables $(1,2, \geqslant 3)$.

\section{The Michigan Longitudinal Study example}

The Michigan Longitudinal Study (MLS) is an ongoing multi-wave prospective study of people at high risk for substance use disorders [27]. The study recruited participants using drunk driving conviction records and door-to-door community canvassing in a four-county area surrounding Michigan's capital city, Lansing. All participants received extensive in-home assessments of their psychiatric symptoms at baseline, and thereafter at 3-year intervals. In order to identify risk factors for AUD at the neighborhood level, we geocoded the residential addresses of the participants and merged the 20 potential covariates derived from census data (listed in Table I) into the MLS database. In our analysis, we included 448 young adult participants ( 72 per cent male), having a mean age of 22 years.

The following is a brief list of the 11 DSM-IV symptom criteria for AUD [28]:

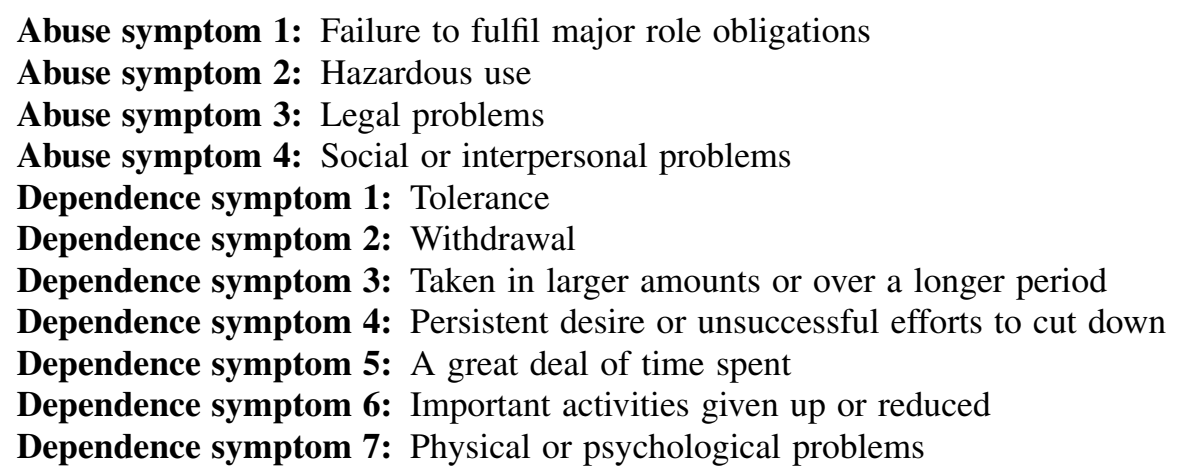

The symptom count (ranges 0-11) serves as an important indicator for AUD severity. As shown in Figure 1, this community sample has fewer zero symptom counts (27 per cent) than the national sample (60 per cent) due to the recruitment protocol targeting the high-risk population. There is also a higher proportion of people with multiple AUD symptoms in this sample. Overall, the zero values in the data are still more than would be predicted from a Poisson regression model. 


\begin{tabular}{|c|c|c|c|c|}
\hline & PR-LASSO & PR-SCAD & ZIP-LASSO & ZIP-SCAD \\
\hline \multicolumn{5}{|c|}{ The Poisson component } \\
\hline Intercept & 0.9788 & 0.9583 & 1.2916 & 1.2575 \\
\hline Covariate \#1 & 0.0022 & 0 & 0 & 0 \\
\hline Covariate \#2 & 0.1554 & 0.2558 & 0 & 0.2205 \\
\hline Covariate \#3 & 0.1028 & 0.2153 & 0.0691 & 0.0878 \\
\hline Covariate \#4 & 0 & 0 & 0 & 0 \\
\hline Covariate \#5 & -0.0192 & 0 & 0 & -0.0440 \\
\hline Covariate \#6 & 0 & 0 & 0 & 0 \\
\hline Covariate \#7 & -0.0789 & -0.0884 & 0 & 0 \\
\hline Covariate \#8 & 0 & 0 & 0 & 0 \\
\hline Covariate \#9 & 0 & -0.2333 & 0 & 0 \\
\hline Covariate \#10 & 0 & 0 & 0 & 0 \\
\hline Covariate \#11 & 0.0313 & 0.0210 & 0 & 0 \\
\hline Covariate \#12 & 0 & 0 & 0 & 0 \\
\hline Covariate \#13 & 0 & 0 & 0 & 0 \\
\hline Covariate \#14 & 0 & 0 & 0 & 0 \\
\hline Covariate \#15 & 0 & 0 & 0 & 0 \\
\hline Covariate \#16 & 0 & 0 & 0 & 0 \\
\hline Covariate \#17 & 0 & 0 & 0 & 0 \\
\hline Covariate \#18 & 0.1224 & 0.2483 & 0 & 0 \\
\hline Covariate \#19 & -0.0676 & -0.1607 & 0 & -0.1926 \\
\hline Covariate \#20 & 0 & 0 & 0 & 0 \\
\hline \multicolumn{5}{|c|}{ The zero component } \\
\hline Intercept & & & -0.7179 & -1.0583 \\
\hline Covariate \#3 & & & & -0.9225 \\
\hline
\end{tabular}

The four competing methods compared in the simulations were used to analyze the MLS data. Table VII shows the estimated regression coefficients. While the Poisson regression methods selected 7-8 covariates, the ZIP methods only selected 1-5 covariates. This may reflect a general finding from the simulations: the Poisson regression methods have a great tendency to over fit the model. Under the SCAD penalty, the Poisson regression model and the ZIP model both identified the high school drop out rate, the unemployment rate, and the disability rate to be associated with the severity of AUD symptomatology. However, the Poisson regression model selected four additional covariates: the proportion of residents with public assistance income, the poverty rate, the proportion of Hispanic residents, and the proportion of residents who did not live at the same address 5 years earlier. Since the primary purpose of involving the variable selection technique for this project was to reduce the neighborhood level covariates, it may not be desirable to adopt the Poisson regression model that resulted in a larger set of covariates, some of which were highly correlated. Furthermore, the Poisson regression model would not have the capacity to specify that the unemployment rate was also associated with the probability of being a drinker.

In order to assess the goodness of fit of the ZIP model, we conducted a series of tests to compare it against alternative models. First, a score test [29] was employed to compare the ZIP model with the Poisson regression model. The result shows that the Poisson regression model is not sufficient to fit the data with excess zeros $\left(\chi^{2}=12.37, \mathrm{df}=1, p<0.001\right)$. We also compared the ZIP model against the saturated model using the Pearson's chi-square statistics implemented in the SAS PROC GENMOD [30] and found the ZIP model fits the data as well as the saturated model $\left(\chi^{2}=436, \mathrm{df}=406, p>0.05\right)$. Moreover, the likelihood ratio test was adopted to compare the reduced ZIP model fitted by each variable selection method against the full ZIP model with all the 20 covariates in both the Poisson and Zero components. The result shows that the reduced ZIP model with only one covariate selected by the ZIP-LASSO may be oversimplified $\left(\chi^{2}=55.06, \mathrm{df}=39, p<0.001\right)$. On the other hand, the reduced ZIP model with the covariates selected by the ZIP-SCAD is sufficient to interpret the neighborhood impact on AUD symptomatology $\left(\chi^{2}=28.08, \mathrm{df}=35, p \approx 0.05\right)$.

In this example, the ZIP model fits the particular data set well. However, in some situations, the ZIP model might not be sufficient to fit the real data. We refer interested readers to recent work by Deng and Paul [31] that developed a series of score tests to facilitate selection among a class of generalized linear models with different link functions, zero-inflation components, and over-dispersion features. 


\section{Discussion}

This study has extended two dominant methods in the recent variable selection literature, the LASSO and the SCAD, to deal with zero-inflated count data that are very common in health surveys. Our simulations demonstrate the danger of using Poisson regression methods to conduct variable selection when excess zeros exist in the data: the methods have a great tendency to over fit the model. The design of our simulations is unique because it preserves the special features of two national databases that have been commonly used in the alcoholism and substance abuse field. As a result, our findings can be easily generalized to the real settings. Our empirical analyses on the data from a community sample not only demonstrate the applications of the methodology but also reflect some trends observed in the simulations.

Based on the results of our simulation on the census data, we recommend the use of ZIP-SCAD in the field of alcoholism and substance abuse research because (I) it can maintain both the specificity and sensitivity at the highest level (mostly over 0.90), (II) it has the lowest MRMSE, and (III) it has the highest value of exact fit. It demonstrates this high level of performance not only in the Poisson component but also in the zero component. In general, its performance improves as the sample size increases, the correlation between covariates decreases, the proportion of non-zero coefficients decreased, and the proportion of zero outcome decreased.

The choice of penalty functions in penalized likelihood has been studied by Fan and Li [10] in depth. They demonstrated that the SCAD penalty improves the LASSO penalty by reducing estimation bias due to the $L_{1}$ penalty, although the LASSO and SCAD share the same spirit in terms of simultaneous variable selection and parameter estimation. The tuning parameter selection is crucial in the penalized likelihood methods. Zhang et al. [19] suggested using the BIC tuning parameter in order for the SCAD to achieve the oracle property. The LASSO with the BIC tuning parameter selector likely yields an estimate with non-ignorable bias. This explains that the MRMSEs of ZIP-LASSO are systematically greater than those for ZIP-SCAD. The BIC tuning parameter selector is a data-driven method. In order to avoid larger estimation bias due to the $L_{1}$ penalty, the BIC tuning parameter selector tends to select a smaller tuning parameter for the ZIP-LASSO than for the ZIP-SCAD. This explains that the ZIP-SCAD estimator has better specificity than the ZIP-LASSO.

Like other variable selection methods [32], the methods proposed in this paper do not work well with small sample sizes. The results of the simulation based on the census data show that for the sample size of 300, the sensitivity level of the ZIP-SCAD only reaches 0.70 in the Poisson component and 0.30 in the zero component. Our simulation with the sample sizes of 100 and 200 (unreported results available upon request) resulted in up to 50 per cent replications failing to converge. Thus, for the case of 40 candidate covariates (20 in the Poisson component and 20 in the zero component), a sample size of 600 would be required for the ZIP-SCAD to perform well. Future studies may evaluate the performance of these variable selection methods under different ratios of the number of candidate covariates to the sample size.

\section{Appendix A: Technical details of implementation of LARS}

For the ease of presentation, we write $\boldsymbol{\theta}=\left(\boldsymbol{\beta}^{\prime}, \boldsymbol{\gamma}^{\prime}\right)^{\prime}$. That is, $\theta_{j}=\beta_{j}$, for $j=1, \ldots, d_{1}$ and $\theta_{j}=\gamma_{j-d_{1}}$, for $j=d_{1}+1, \ldots, d_{1}+d_{2}$. Similarly, $\boldsymbol{\theta}^{(0)}=\left(\boldsymbol{\beta}^{(0)^{\prime}}, \boldsymbol{\gamma}^{(0)^{\prime}}\right)^{\prime}$. Denote

$$
\Sigma_{0}=-\nabla^{2} \ell\left(\boldsymbol{\beta}^{(0)}, \quad \gamma^{(0)}\right)=-\nabla^{2} \ell\left(\boldsymbol{\theta}^{(0)}\right) .
$$

In what follows, we give the details on how to employ the LARS algorithm [16] to the one-step sparse estimator $\boldsymbol{\theta}^{(1)}$, given the initial value $\boldsymbol{\theta}^{(0)}$.

Step 1: Define index sets

$$
U=\left\{j: p_{a_{j}}^{\prime}\left(\left|\beta_{j}^{(0)}\right|\right)=0\right\} \cup\left\{d_{1}+k: p_{b_{k}}^{\prime}\left(\left|\gamma_{k}^{(0)}\right|\right)=0\right\},
$$

and

$$
V=\left\{j: p_{a_{j}}^{\prime}\left(\left|\beta_{j}^{(0)}\right|\right) \neq 0\right\} \cup\left\{d_{1}+k: p_{b_{k}}^{\prime}\left(\left|\gamma_{k}^{(0)}\right|\right) \neq 0\right\}
$$




\section{Statistics}

Step 2: Find the Cholesky decomposition of $\Sigma_{0}$. That is, to find a $\left(d_{1}+d_{2}\right) \times\left(d_{1}+d_{2}\right)$ matrix $L$, such that,

$$
\Sigma_{0}=L^{\prime} L
$$

Create working data by

$$
Y^{*}=L \boldsymbol{\theta}^{(0)},
$$

let $\mathbf{x}_{j}^{*}=\tau / n p_{\tau_{j}}^{\prime}\left(\left|\theta_{j}^{(0)}\right|\right) \ell_{j}^{*}$ for $j \in V$, where $\ell_{j}^{*}$ is the $j$ th column of $L, \tau_{j}=a_{j}$ for $j \leqslant d_{1}$ and $\tau_{j}=b_{j-d_{1}}$ for $j>d_{1}$. Further write

$$
X^{*}=\left[X_{U}^{*}, X_{V}^{*}\right], \quad \boldsymbol{\theta}^{(1)}=\left(\boldsymbol{\theta}_{U}^{(1)^{\prime}}, \boldsymbol{\theta}_{V}^{(1)^{\prime}}\right)^{\prime} .
$$

Step 3: Let $H_{U}$ be the projection matrix in the space of $X_{U}^{*}$, i.e. $H_{U}=X_{U}^{*}\left(X_{U}^{*^{\prime}} X_{U}^{*}\right)^{-} X_{U}^{*^{\prime}}$. Compute

$$
Y^{* *}=Y^{*}-H_{U} Y^{*}, \quad X_{V}^{* *}=X_{V}^{*}-H_{U} X_{V}^{*}
$$

Step 4: Apply the LARS algorithm to solve

$$
\hat{\boldsymbol{\theta}}_{V}^{*}=\arg \min _{\boldsymbol{\theta}}\left\{\frac{1}{2}\left\|Y^{* *}-X_{V}^{* *} \boldsymbol{\theta}\right\|+\tau \sum_{j=1}^{d_{1}+d_{2}}\left|\theta_{j}\right|\right\} .
$$

Step 5: Compute $\hat{\boldsymbol{\theta}}_{U}^{*}=\left(X_{U}^{*^{\prime}} X_{U}^{*}\right)^{-1} X_{U}^{*^{\prime}}\left(Y^{*}-X_{V}^{*} \hat{\boldsymbol{\theta}}_{V}^{*}\right)$.

It follows that the one-step LLA estimator is:

$$
\hat{\boldsymbol{\theta}}_{U}^{(1)}=\hat{\boldsymbol{\theta}}_{U}^{*} \quad \text { and } \quad \hat{\theta}_{j}^{(1)}=\frac{\tau}{n p_{\tau_{j}}^{\prime}\left(\left|\theta_{j}^{(0)}\right|\right)} \hat{\theta}_{j}^{*} \quad \text { for } j \in V .
$$

\section{Appendix B: Regularity conditions and key derivation of oracle property of ZIP with one-step SCAD}

We will need the following regularity conditions, under which the oracle property of the one-step SCAD for ZIP may be established.

\section{B.1. Regularity conditions}

(A) The observations $\mathbf{v}_{i}=\left\{\boldsymbol{w}_{i}, y_{i}\right\}, i=1, \cdots, n$, is an independent and identically distributed sample from the ZIP model (1). Denote $\boldsymbol{\theta}=\left(\boldsymbol{\beta}^{\prime}, \boldsymbol{\gamma}^{\prime}\right)^{\prime}$ and $\ell\left(\boldsymbol{\theta}, \mathbf{v}_{i}\right)$ to be the log-likelihood function of the $i$ th observation $\mathbf{v}_{i}$. Assume that the first and second partial derivatives $\ell\left(\boldsymbol{\theta}, \mathbf{v}_{i}\right)$ with respect to $\theta_{j}$ satisfies the equations

$$
E_{\boldsymbol{\theta}}\left[\frac{\partial \ell\left(\boldsymbol{\theta}, \mathbf{v}_{i}\right)}{\partial \theta_{j}}\right]=0
$$

and

$$
I_{j k}(\boldsymbol{\theta})=E_{\boldsymbol{\theta}}\left[\frac{\partial}{\partial \theta_{j}} \ell\left(\boldsymbol{\theta}, \mathbf{v}_{i}\right) \frac{\partial}{\partial \theta_{k}} \ell\left(\boldsymbol{\theta}, \mathbf{v}_{i}\right)\right]=E_{\boldsymbol{\theta}}\left[-\frac{\partial^{2}}{\partial \theta_{j} \partial \theta_{k}} \ell\left(\boldsymbol{\theta}, \mathbf{v}_{i}\right)\right] .
$$

for $j=1, \cdots, d_{1}+d_{2}$.

(B) The Fisher information matrix

$$
I(\boldsymbol{\theta})=E\left\{\left[\frac{\partial}{\partial \boldsymbol{\theta}} \ell\left(\boldsymbol{\theta}, \mathbf{v}_{i}\right)\right]\left[\frac{\partial}{\partial \boldsymbol{\theta}} \ell\left(\boldsymbol{\theta}, \mathbf{v}_{i}\right)\right]^{\prime}\right\}
$$

is finite and positive definite at $\boldsymbol{\theta}=\boldsymbol{\theta}_{0}$. 
(C) There exists an open subset $\omega$ of $\Omega$ containing the true parameter point $\boldsymbol{\theta}_{0}$ such that for almost all $\mathbf{v}_{i}, \ell\left(\boldsymbol{\theta}, \mathbf{v}_{i}\right)$ admits all third derivatives $\partial \ell\left(\boldsymbol{\theta}, \mathbf{v}_{i}\right) / \partial \theta_{j} \partial \theta_{k} \partial \theta_{l}$ for all $\boldsymbol{\theta} \in \omega$. Further there exist functions $M_{j k l}$ such that

$$
\left|\frac{\partial^{3}}{\partial \beta_{j} \partial \beta_{k} \partial \beta_{l}} \ell\left(\boldsymbol{\theta}, \mathbf{v}_{i}\right)\right| \leqslant M_{j k l}\left(\mathbf{v}_{i}\right) \text { for all } \boldsymbol{\theta} \in \omega,
$$

where $m_{j k l}=E_{\boldsymbol{\theta}_{0}}\left[M_{j k l}\left(\mathbf{v}_{i}\right)\right]<\infty$ for $j, k, l$.

These regularity conditions guarantee asymptotic normality of the ordinary maximum likelihood estimate of the ZIP model. See, for example, Lehmann and Casella [33].

\section{B.2. Key derivation of oracle properties of the one-step SCAD estimator}

Under Conditions (A)-(C), we can show that the maximum likelihood estimate of $\boldsymbol{\theta}$ in the ZIP model, denoted by $\hat{\boldsymbol{\theta}}(\mathrm{mle})$, is root $n$ consistent,

$$
-\left.\frac{1}{n} \sum_{i=1}^{n} \frac{\partial^{2}}{\partial \boldsymbol{\theta} \partial \boldsymbol{\theta}^{\prime}} \ell\left(\boldsymbol{\theta}, \mathbf{v}_{i}\right)\right|_{\boldsymbol{\theta}=\hat{\boldsymbol{\theta}}(\mathrm{mle})} \rightarrow I\left(\boldsymbol{\theta}_{0}\right)
$$

in probability as $n \rightarrow \infty$, and

$$
\sqrt{n}\left(\hat{\boldsymbol{\theta}}(\mathrm{mle})-\boldsymbol{\theta}_{0}\right) \rightarrow N\left(0, I^{-1}\left(\boldsymbol{\theta}_{0}\right)\right)
$$

in distribution as $n \rightarrow \infty$. As a direct application of Theorem 5 of Zou and Li [12], if $\lambda_{n} \rightarrow 0$ and $\sqrt{n} \lambda_{n} \rightarrow \infty$, then the one-step SCAD estimator for the ZIP model possesses the oracle property: with probability tending to one, the estimate for zero coefficients equals 0 , and the estimate for nonzero coefficient has an asymptotic normal distribution with mean being the true value of nonzero coefficient and variance $I_{1}^{-1}\left(\theta_{0}\right)$, where $I_{1}\left(\theta_{0}\right)$ is the submatrix of the Fisher information matrix corresponding to the nonzero coefficients.

\section{Acknowledgements}

Buu's research was supported by a National Institutes of Health (NIH) grant, K01 AA16591; Li's research was supported by a NIH grant, R21 DA024260 and a National Science Foundation (NSF) grant DMS 0348869; and Tan's research was supported by a NIH grant P50 DA10075. The content is solely the responsibility of the authors and does not necessarily represent the official views of the NIH, NSF, or the U.S. Census Bureau. The authors thank Dr Robert A. Zucker for his permission to use the data of Michigan Longitudinal Study (MLS) as an example in this manuscript. The data collection of the MLS was supported by a NIH grant R37 AA07065 awarded to Dr Zucker.

\section{References}

1. Buu A, DiPiazza C, Wang J, Puttler LI, Fitzgerald HE, Zucker RA. Parent, family, and neighborhood effects on the development of child substance use and other psychopathology from preschool to the start of adulthood. Journal of Studies on Alcohol and Drugs 2009; 70:489-498.

2. Cerda M, Sanchez BN, Galea S, Tracy M, Buka SL. Estimating co-occurring behavioral trajectories within a neighborhood context. American Journal of Epidemiology 2008; 168(10):1190-1203. DOI: 10.1093/aje/kwn241.

3. Luthar SS, Cushing G. Neighborhood influences and child development: a prospective study of substance abusers' offspring. Development and Psychopathology 1999; 11(4):763-784. DOI: 10.1017/S095457949900231X.

4. Saxe L, Kadushin C, Beveridge A, Livert D, Tighe E, Rindskopf D, Ford J, Brodky A. The visibility of illicit drugs: implications for community-based drug control strategies. American Journal of Public Health 2001; 91(12):1987-1994. DOI: $10.2105 /$ AJPH.91.12.1987.

5. Tarter RE, Kirisci L, Gavaler JS, Reynolds M, Kirillova G, Clark DB, Wu J, Moss HB, Vanyukov M. Prospective study of the association between abandoned dwellings and testosterone level on the development of behaviors leading to cannabis use disorder in boys. Biological Psychiatry 2009; 65:116-121. DOI: 10.1016/j.biopsych.2008.08.032.

6. Akaike H. A new look at the statistical model identification. IEEE Transactions on Automatic Control 1974; 19:716-723.

7. Schwarz G. Estimating the dimension of a model. Annals of Statistics 1978; 6:461-464.

8. Breiman L. Better subset regression using the nonnegative garrote. Technometrics 1995; 37:373-384.

9. Tibshirani RJ. Regression shrinkage and selection via the LASSO. Journal of Royal Statistical Society B 1996; 58:267-288.

10. Fan J, Li R. Variable selection via nonconcave penalized likelihood and its oracle properties. Journal of American Statistical Association 2001; 96:1348-1360. 
11. Park MY, Hastie T. L1-regularization path algorithm for generalized linear models. Journal of Royal Statistical Society B 2007; 69:659-677.

12. Zou H, Li R. One-step sparse estimates in nonconcave penalized likelihood models (with Discussion). Annals of Statistics 2008; 36:1509-1566. DOI: 10.1214/009053607000000802.

13. Lambert D. Zero-inflated Poisson regression, with an application to defects in manufacturing. Technometrics 1992; 34:1-13.

14. Dean BB, Calimlim BM, Kindermann SL, Khandker RK, Tinkelman D. The impact of uncontrolled asthma on absenteeism and health-related quality of life. Journal of Asthma 2009; 46:861-866. DOI: 10.3109/02770900903184237.

15. Min Y, Agresti A. Random effect models for repeated measures of zero-inflated count data. Statistical Modeling 2005; 5:1-19.

16. Efron B, Hastie T, Johnstone I, Tibshirani R. Least angle regression. The Annals of Statistics 2004; 32:407-499.

17. Wang H, Leng C. Unified Lasso estimation via least squares approximation. Journal of the American Statistical Association 2007; 102:1039-1048.

18. Fan J, Li R. New estimation and model selection procedures for semiparametric modeling in longitudinal data analysis. Journal of the American Statistical Association 2004; 99:710-723. DOI: 10.1198/0162145040000001060.

19. Zhang Y, Li R, Tsai CL. Regularization parameter selections via generalized information criterion. Journal of the American Statistical Association 2010; 105:312-323. DOI: 10.1198/jasa.2009.tm08013.

20. Burton A, Altman DG, Royston P, Holder RL. The design of simulation studies in medical statistics. Statistics in Medicine 2006; 25:4279-4292. DOI: 10.1002/sim.2673.

21. Grant BF, Stinson FS, Dawson DA, Chou SP, Dufour MC, Compton W, Pickering RP, Kaplan K. Prevalence and co-occurrence of substance use disorders and independent mood and anxiety disorders: results from the National Epidemiologic Survey on Alcohol and Related Conditions. Archives of General Psychiatry 2004; 61:807-816. DOI: 10.1001/archpsyc.61.8.807.

22. Chassin L, Barrera M, Bech K, Kossak-Fuller J. Recruiting a community sample of adolescent children of alcoholics: a comparison of three subject sources. Journal of Studies on Alcohol 1992; 53:316-319.

23. Clark DB, Kirisci L, Mezzich A, Chung T. Parental supervision and alcohol use in adolescence: developmentally specific interactions. Journal of Developmental and Behavioral Pediatrics 2008; 29:285-292.

24. Hawkins JD, Kosterman R, Catalano RF, Hill KG, Abbott RD. Promoting positive adult functioning through social development intervention in childhood: long-term effects from the Seattle Social Development Project. Archives of Pediatrics and Adolescent Medicine 2005; 159:25-31.

25. Iacono WG, Carlson SR, Taylor J, Elkins IJ, McGue M. Behavioral disinhibition and the development of substance-use disorders: findings from the Minnesota Twin Family Study. Development and Psychopathology 1999; 11:869-900. DOI: $10.1017 /$ S0954579499002369.

26. Windle M, Mun EY, Windle RC. Adolescent-to-young adulthood heavy drinking trajectories and their prospective predictors. Journal of Studies on Alcohol 2005; 66:313-322.

27. Zucker RA, Fitzgerald HE, Refior SK, Puttler LI, Pallas DM, Ellis DA. The clinical and social ecology of childhood for children of alcoholics: description of a study and implications for a differentiated social policy. In Children of Addiction, Fitzgerald HE, Lester BM, Zuckerman BS (eds). Garland Press: New York, 2000; 109-141.

28. American Psychiatric Association. Diagnostic and Statistical Manual (4th edn). American Psychiatric Association: Washington, DC, 1994.

29. van den Broek J. A score test for zero inflation in a Poisson distribution. Biometrics 1995; 51:738-743.

30. SAS Institute Inc. SAS/STAT 9.2 User's Guide. SAS Institute Inc.: Cary, NC, 2008.

31. Deng D, Paul SR. Score tests for zero-inflation and over-dispersion in generalized linear models. Statistica Sinica 2005; 15:257-276.

32. Wiegand RE. Performance of using multiple stepwise algorithms for variable selection. Statistics in Medicine 2010; 29:1647-1659.

33. Lehmann E, Casella G. Theory of Point Estimation (2nd edn). Springer: Berlin, 1998. 